\title{
Second-look endoscopy findings after endoscopic submucosal dissection for colorectal epithelial neoplasms
}

\author{
Soo-kyung Park ${ }^{1, \star}$, Hyeon Jeong Goong ${ }^{2, \star}$, Bong Min $\mathrm{Ko}^{2}$, Haewon Kim² ${ }^{2}$, Hyo Sun Seok ${ }^{3}$, and \\ Moon Sung Lee ${ }^{2}$
}

\begin{abstract}
${ }^{1}$ Division of Gastroenterology, Department of Internal Medicine and Gastrointestinal Cancer Center, Kangbuk Samsung Hospital,

Sungkyunkwan University School of Medicine, Seoul; ${ }^{2}$ Digestive Disease Center and Research Institute and Department of Internal Medicine, Soonchunhyang University School of Medicine, Bucheon; ${ }^{3}$ Total Healthcare Center, Kangbuk Samsung Hospital, Sungkyunkwan University School of Medicine, Seoul, Korea
\end{abstract}

Received: February 14, 2020

Revised : March 13, 2020

Accepted: April 10, 2020

Correspondence to

Bong Min Ko, M.D.

Digestive Disease Center

and Research Institute,

Soonchunhyang University

Bucheon Hospital, 170 Jomaru-

ro, Wonmi-gu, Bucheon 14584,

Korea

Tel: $+82-32-621-5094$

Fax: +82-32-621-5080

E-mail: kopa9445@schmc.ac.kr https://orcid.org/0000-0002-

o635-4454

*These authors contributed equally to this work.
Background/Aims: Although second-look endoscopy (SLE) is frequently performed after gastric endoscopic submucosal dissection (ESD) to prevent bleeding, no studies have reported SLE findings after colorectal ESD. This study aimed to investigate SLE findings and their role in preventing delayed bleeding after colorectal ESD.

Methods: Post-ESD ulcer appearances were divided into coagulation (with or without remnant minor vessels) and clip closure groups. SLE findings were categorized according to the Forrest classification (high-risk ulcer stigma [type I and IIa] and low-risk ulcer stigma [type IIb, IIc, III, or clip closure]), and risk factors for high-risk ulcer stigma were analyzed.

Results: Among the 375 cases investigated, SLEs were performed in 171 (45.6\%) patients. The incidences of high-risk ulcer stigma and low-risk stigma were 5.3\% (9/171) and 94.7\% (162/171), respectively. During SLE, endoscopic hemostasis was performed more frequently in the high-risk ulcer stigma group than in the lowrisk ulcer stigma group (44.4\% [4/9] vs. 1.9\% [3/162], respectively; $p<0.001)$, but most of the endoscopic hemostasis in the high-risk ulcer stigma group $(3 / 4,75.0 \%)$ were prophylactic hemostasis. Post-ESD delayed bleeding occurred in three (o.8\%) patients belonging to the SLE group, of which, one patient was from the high-risk stigma group and two were from the low-risk stigma group.

Conclusions: The incidence of high-risk ulcer stigma during SLE was low, and delayed bleeding occurred in, both, high-risk and low-risk groups of SLE. SLEs performed after colorectal ESD may not be effective in preventing delayed bleeding, and further prospective studies are needed to evaluate the efficacy of SLE in post-colorectal ESD.

Keywords: Colorectal neoplasms; Endoscopic mucosal resection; Second-look endoscopy

\section{INTRODUCTION}

Colorectal endoscopic submucosal dissection (ESD) has recently been performed to treat large colorectal epi- thelial neoplasms. Recent studies have reported favorable long-term outcomes of ESD, with 100\% 5-year disease-specific survival and $1.5 \%$ to $3.8 \%$ local recurrence rates $[1,2]$. However, colorectal ESD is still a technically 
difficult and time-consuming procedure because of the anatomical characteristics and peristalsis of the colon. The adverse events most significantly related to patients' morbidity and mortality are bleeding and perforation. Reports have revealed the incidence of post-ESD bleeding to be $0 \%$ to $12 \%$ [3-5]. Although most cases can be managed endoscopically, post-ESD bleeding may result in surgery or the requirement for intensive care. Therefore, several studies have tried to evaluate the risk factors of post-ESD bleeding $[6,7]$. Furthermore, studies have recently suggested that the appearance of mucosal defects after mucosal resections may predict post-procedural bleeding [8-10].

Prospective, randomized trials have revealed the efficacy of second-look endoscopy (SLE) performed after endoscopic hemostasis for bleeding peptic ulcers [11-13]. Thus, SLE was routinely performed 1 day after gastric ESD to detect and evaluate the risk of post-ESD bleeding. However, recent randomized controlled trials and meta-analyses suggest that SLE does not prevent delayed bleeding after gastric ESD [14-16]. Moreover, the Forrest classification of post-ESD ulcer stigma on SLE was not significantly different between patients with or without delayed bleeding [17]. Therefore, experts recommend that SLE be performed only in cases with clinical evidence of recurrent bleeding, poor visualization at the index endoscopy, or an endoscopist's suspicion of inadequate hemostasis. However, no study has demonstrated the findings and efficacy of SLE performed after colorectal ESD. Thus, we aimed to investigate SLE findings and the role of SLE in preventing delayed bleeding after colorectal ESD.

\section{METHODS}

\section{Patients}

We enrolled a total of 426 lesions of 426 patients diagnosed with colorectal epithelial neoplasia and subsequently treated with ESD at Soonchunhyang University Bucheon Hospital, Bucheon, Korea between March 2010 and September 2017. We reviewed the medical records and endoscopic images of all patients. Indications of ESD were based on the Colorectal ESD Standardization Implementation Working Group guidelines [18]. Patients who were not followed up 1 month post-ESD or had post-ESD ulcer images that were not assessable during SLE were excluded. This study was performed in accordance with the ethical principles of the Declaration of Helsinki and was approved by the Institutional Review Board at Soonchunhyang University Bucheon Hospital (IRB No., SCHBC 2018-07-025). All patients provided written informed consent before the procedure.

\section{Colorectal ESD}

The patients underwent ESD under conscious sedation using midazolam and propofol. Antiplatelet and anticoagulant medications were discontinued and restarted following the American Society for Gastrointestinal Endoscopy (ASGE) guidelines [19]. Patients were instructed to discontinue antiplatelet therapy 7 days and anticoagulant medications 3 to 5 days before the procedure. Two to four liters of polyethylene glycol were used for bowel preparation. ESD was performed at least 4 to 6 hours after polyethylene glycol ingestion. Two expert endoscopists, certified by the Korean Society of Gastrointestinal Endoscopy, performed the colorectal ESDs. All procedures were performed using colonoscopes (CF-Q240AL, CF-H26oAL, PCF-Q26oJI, Olympus Optical Co., Tokyo, Japan) with a disposable cap on its tip. The colorectal ESD process was as follows. (1) Before ESD, the depth of invasion was evaluated using narrowband imaging or magnifying chromoendoscopy with indigo carmine (0.4\%) or crystal violet (0.05\%) solutions. (2) We used sodium hyaluronate (Endo-Ease, Unimed Co., Seoul, Korea) and epinephrine-normal saline solution (1:100,000) for submucosal injection. (3) After submucosal injection, the mucosa around the neoplasms was circumferentially incised. The Hook knife (KD-62oLR, Olympus Medical Systems, Tokyo, Japan), Flex knife (KD-63oL, Olympus Medical Systems), or DualKnife (KD-65oL, Olympus Medical Systems) was used for cutting the margins. The Intelligent Cut and Coagulation system (ERBE, Tübingen, Germany) was used as a power source. The cut (VIO 300, ERBE; Endo Cut Q mode, effect 3) and coagulation current (VIO 300, Forced Coagulation mode) were applied during ESD. (4) After cutting the margins, submucosal dissection was performed using the same knife used for cutting the margins. 


\section{Hemostasis during ESD}

To prevent hemorrhages during ESD, when a vessel $<2$ $\mathrm{mm}$ in diameter (minor vessel) was detected in the submucosa, it was cut with a knife in coagulation mode (e.g., Forced Coagulation $40 \mathrm{~W}$, effect 3 in VIO $300 \mathrm{D})$. When a vessel $\geq 2 \mathrm{~mm}$ in diameter (major vessel) was detected, hemostat forceps (e.g., Coagrasper; FD-41oLR, Olympus Optical Co.) were used in soft coagulation mode (output $60 \mathrm{~W}$, effect 5 in VIO 300 D). Endoscopic hemostasis was achieved using hemostatic forceps whenever active bleeding occurred. Hemostatic clips (EZ clip, Olympus Medical Systems) were used when hemostasis could not be achieved using hemostatic forceps.

After neoplasm dissection, the base of artificial ulcers was closely evaluated, and prophylactic hemostasis was performed using clip closures or hemostatic forceps coagulation. Clip closure was defined as a completely sutured mucosal defect using endoclips and performed for deep ulcers. In other cases, all major vessels and some minor vessels were coagulated using hemostatic forceps. In this study, we retrospectively reviewed several images of the ulcer base using a high-definition endoscope. The post-ESD ulcer appearance was divided into a clip closure group and a coagulation group. The coagulation group was further divided into visible vessel (with remnant minor vessels) and non-visible vessel (without remnant minor vessels) groups (Fig. 1). The artificial ulcers mainly managed by hemostatic forceps coagulation with local hemostasis by endoclips were classified into the coagulation group.

\section{SLE performed after ESD and post-procedural treatment}

The SLE group underwent endoscopy on day 1 postESD to evaluate post-ESD ulcer stigma. We did not perform additional bowel preparation before SLE. The SLE process was performed depending on the endoscopist's decision, but the practical policy of our endoscopy center was as follows. Endoscopic hemostasis was considered if high-risk lesions, such as those that were oozing or with vessels visible in the ulcer bed, were observed. Hemostasis was achieved using hemostatic forceps, and hemostatic clips were used when hemostasis could not be achieved using hemostatic forceps. Prophylactic hemostasis was performed using hemostatic forceps coagulation for major vessels and argon plasma coagulation
(APC) for minor vessels. After confirmation of lesion status, patients started taking sips of water and light meals on day 2. If hemostasis was achieved during the SLE, patients started taking sips of water on day 3 and light meals on day 4. In this study, SLE findings were retrospectively reviewed and categorized according to the Forrest classifications: types Ia (spurting), Ib (oozing), IIa (visible nonbleeding vessel), IIb (adherent clot), IIc (hematin on ulcer base), and III (clean base) (Supplementary Fig. 1). We classified types I and IIa into a high-risk ulcer stigma group. Types IIb, IIc, and III and clip closure were classified into a low-risk ulcer stigma group.

In patients who did not undergo SLE, if laboratory and clinical findings on day 1 did not show post-ESD bleeding, patients started on sips of water and light meal on day 2. All patients visited the outpatient department to confirm their final pathological results within 3 weeks after discharge.

\section{Data collection and outcome measures}

Patient-related factors, including age, sex, and the use of antiplatelet or anticoagulant medications, were reviewed. Lesion-related factors, including the size, location, gross morphology, and histopathology, were evaluated using endoscopic records, images, and histopathological reports. Procedure-related factors, including procedural time (from the beginning of circumferential margin cutting to the end of dissection) and post-ESD ulcer appearance, were documented. We defined post-ESD delayed bleeding as hematochezia or decrease in hemoglobin level of $\leq 2 \mathrm{~g} / \mathrm{dL}$ from 24 hours to 1 month post-ESD.

The primary outcome measures of this study were the SLE findings and rate of hemostasis during SLE. Secondary outcome measures were risk factors for highrisk ulcer stigma in SLE and the rate of delayed bleeding.

\section{Statistical analysis}

Continuous variables are presented as mean \pm SD. Categorical variables are presented as numbers and percentages. Differences in the baseline characteristics between each group were compared using the chi-square test for categorical variables and Student's $t$ test for continuous variables. Fisher's exact test was used when the expected values in any of the cells of a contingency table are below 5. The $p$ values $<0.05$ were considered statistically 


\section{KJIM}
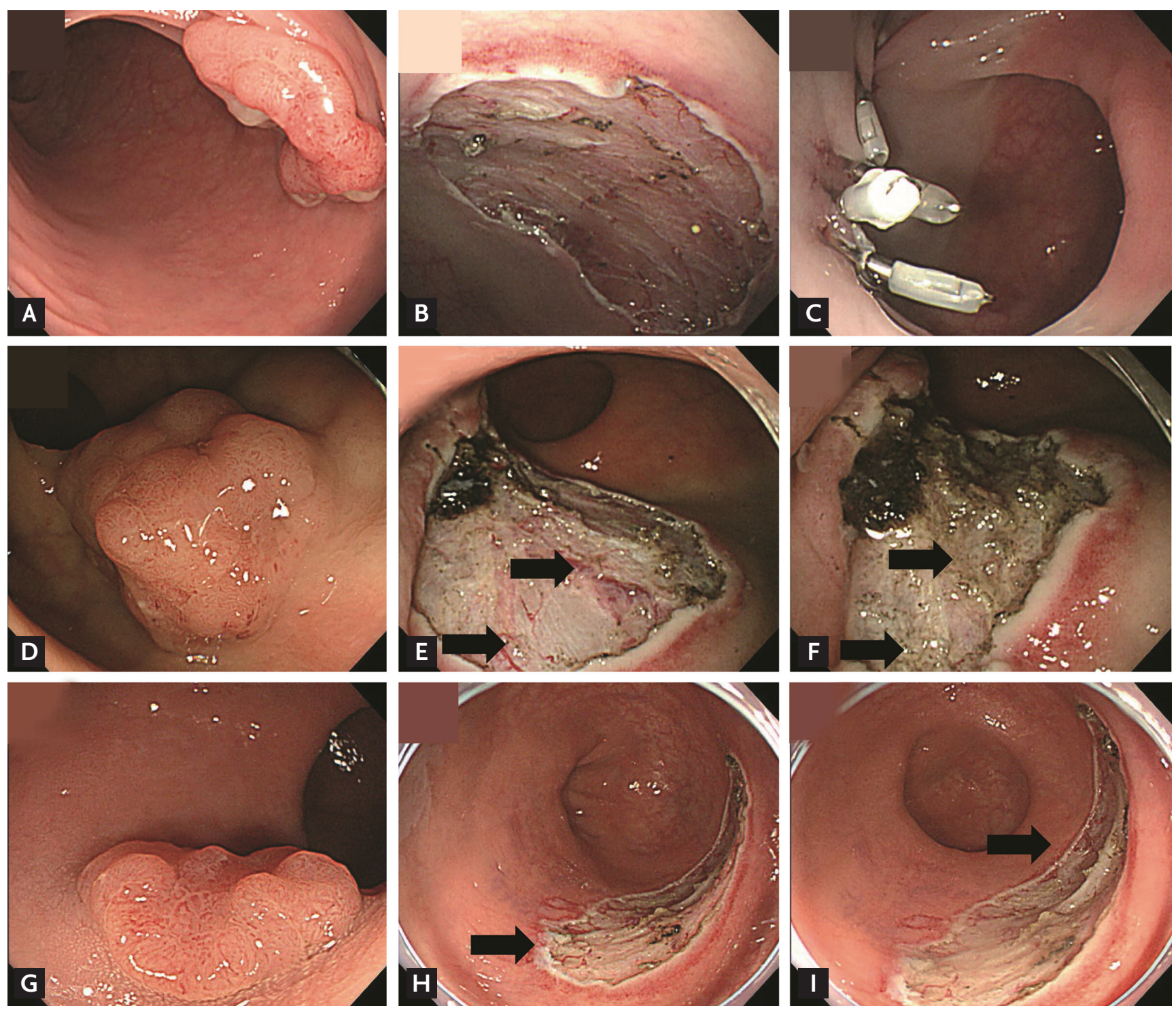

Figure 1. The appearance of artificial ulcers after the completion of endoscopic submucosal dissection. (A, B, C) An Isp type lesion was dissected, then the whole artificial ulcer was completely closed with hemoclips (clip closure group). (D, E, F) After the dissection of the Isp lesion, prophylactic coagulation was done for major and minor vessels (arrows; coagulation, non-visible vessel group). (G, H, I) After the dissection of the Isp type lesion, there were remnant minor vessels on the artificial ulcer (arrows; coagulation, visible vessel group).

significant. IBM SPSS Statistics version 20.0 (IBM Corp., Armonk, NY, USA) was used for statistical analyses.

\section{RESULTS}

\section{Baseline characteristics of patients and colorectal neoplasms}

Among the 426 patients, 30 did not visit the outpatient department within 1 month and were excluded. More- over, 21 SLE patients with unevaluated post-ESD ulcers at the proximal colon, due to the procedure being finished after confirming the absence of remnant blood clots or fresh blood in the sigmoid colon or rectum, were also excluded. Therefore, 375 colorectal epithelial neoplasms in 375 patients (SLE group, $\mathrm{n}=171$; no-SLE group, $\mathrm{n}=204$ ) were included in the final analysis. Patient and neoplasm characteristic differences between each group are described in Table 1 . There were no significant differences between the SLE and no-SLE groups 
Table 1. Baseline characteristics of patients and the colorectal polyps in the SLE and no-SLE groups

\begin{tabular}{|c|c|c|c|c|}
\hline \multirow{2}{*}{ Variable } & \multicolumn{4}{|c|}{ Group } \\
\hline & Total $(\mathrm{n}=375)$ & $\operatorname{SLE}(\mathrm{n}=171)$ & No-SLE $(\mathrm{n}=204)$ & $p$ value \\
\hline \multicolumn{5}{|l|}{ Patient-related factor } \\
\hline Age, year & $61.3 \pm 11.8$ & $61.7 \pm 11.2$ & $60.9 \pm 12.3$ & 0.46 \\
\hline Male sex & $205(54.7)$ & $84(49.1)$ & $121(59 \cdot 3)$ & 0.06 \\
\hline Drug use $^{a}$ & $45(12.0)$ & $22(12.9)$ & $23(11.3)$ & 0.63 \\
\hline Antiplatelet & $44(11.7)$ & $22(12.9)$ & $22(10.8)$ & \\
\hline Aspirin & $37(9 \cdot 9)$ & $21(12.3)$ & $16(7.8)$ & \\
\hline Clopidogrel & $3(0.8)$ & o & $3(1.5)$ & \\
\hline Cilostazol & $1(0.3)$ & 0 & $1(0.5)$ & \\
\hline Aspirin + clopidogrel & $3(0.8)$ & $1(0.6)$ & $2(1)$ & \\
\hline Anticoagulant (warfarin) & $1(0.3)$ & 0 & $1(0.5)$ & \\
\hline \multicolumn{5}{|l|}{ Lesion-related factor } \\
\hline Size, $\mathrm{mm}$ & $32.4 \pm 14.4$ & $32.0 \pm 15.2$ & $32.7 \pm 13.6$ & 0.65 \\
\hline Location & & & & 0.003 \\
\hline Ascending/transverse colon & $115(30.7)$ & $38(22.2)$ & $77(37.7)$ & \\
\hline Descending/sigmoid colon & $96(25.6)$ & $37(21.6)$ & $59(28.9)$ & \\
\hline Rectum & $164(43 \cdot 7)$ & $96(56.1)$ & $68(33 \cdot 3)$ & \\
\hline Morphology & & & & 0.03 \\
\hline LST-G & $133(35 \cdot 5)$ & $72(42.1)$ & $61(29.9)$ & \\
\hline LST-NG & $149(39 \cdot 7)$ & $55(32.2)$ & $94(46.1)$ & \\
\hline Polypoid & $93(24.8)$ & $44(25 \cdot 7)$ & $49(24.0)$ & \\
\hline Histopathology & & & & 0.03 \\
\hline Low grade dysplasia & $126(33.6)$ & $53(31.0)$ & $73(35.8)$ & \\
\hline High grade dysplasia & $95(25 \cdot 3)$ & $39(22.8)$ & $56(27 \cdot 5)$ & \\
\hline Adenocarcinoma-M/SMs ${ }^{\mathrm{b}}$ & $126(33 \cdot 6)$ & $63(36.8)$ & $63(30.9)$ & \\
\hline Adenocarcinoma-SM/d & $28(7.5)$ & $16(9.4)$ & $12(5 \cdot 9)$ & \\
\hline \multicolumn{5}{|l|}{ Procedure-related factor } \\
\hline Procedural time, min & $72.3 \pm 67.3$ & $69.7 \pm 63.0$ & $74.5 \pm 70.8$ & 0.08 \\
\hline Post-ESD ulcer appearance & & & & 0.03 \\
\hline Clip closure & $94(25.1)$ & $52(30.4)$ & $42(20.6)$ & \\
\hline Coagulation & $281(74.9)$ & $119(69.6)$ & $162(79.4)$ & \\
\hline Visible vessel & $190(50.7)$ & $68(39.8)$ & $122(59.8)$ & \\
\hline Non-visible vessel & $91(24.3)$ & $51(29.8)$ & $40(19.6)$ & \\
\hline
\end{tabular}

Values are presented as mean $\pm \mathrm{SD}$ or number (\%).

SLE, second-look endoscopy; LST-G, lateral spreading tumor-granular type; LST-NG, lateral spreading tumor-nongranular type; M/SMs, mucosa to superficial submucosa invasion (<1,000 $\mu \mathrm{m})$; SM/d, deep submucosa invasion $(\geq 1,000 \mu \mathrm{m})$; ESD, endoscopic submucosal dissection.

aDrug use was categorized as "use" or "no use" for statistical analysis using chi-square test.

in terms of patient-related factors, including age, sex, and current use of antiplatelet or anticoagulant agents. Regarding lesion-related factors, the proportion of proximal and lateral spreading tumor-nongranular type neoplasms was higher in the no-SLE group. In addition, the proportion of adenocarcinoma-SM/day was higher 
Table 2. Risk factors for high-risk ulcer stigma on the second-look endoscopy

\begin{tabular}{|c|c|c|c|}
\hline Variable & $\begin{array}{l}\text { High-risk ulcer stigma } \\
\qquad(\mathrm{n}=9)\end{array}$ & $\begin{array}{l}\text { Low-risk ulcer stigma } \\
\qquad(\mathrm{n}=162)\end{array}$ & $p$ value \\
\hline \multicolumn{4}{|l|}{ Patient-related factor } \\
\hline Age, year & $58.8 \pm 11.7$ & $61.9 \pm 11.2$ & 0.41 \\
\hline Use of antiplatelet/anticoagulant & $1(11.1)$ & $22(13.6)$ & 0.65 \\
\hline \multicolumn{4}{|l|}{ Lesion-related factor } \\
\hline Location & & & 0.24 \\
\hline Ascending/transverse colon & $2(22.2)$ & $36(22.2)$ & \\
\hline Descending/sigmoid colon & 0 & $37(22.8)$ & \\
\hline Rectum & $7(77.8)$ & $89(54.9)$ & \\
\hline Size, $\mathrm{mm}$ & & & 0.99 \\
\hline$<40$ & $7(77.8)$ & $120(74.1)$ & \\
\hline$\geq 40$ & $2(22.2)$ & $42(25 \cdot 9)$ & \\
\hline Morphology & & & 0.97 \\
\hline LST-G & $4(44 \cdot 4)$ & $68(42.0)$ & \\
\hline LST-NG & $3(33 \cdot 3)$ & $52(32.1)$ & \\
\hline Polypoid & $2(22.2)$ & $42(25 \cdot 9)$ & \\
\hline Histopathology & & & 0.62 \\
\hline Low/high grade dysplasia & $3(33 \cdot 3)$ & $89(54.9)$ & \\
\hline Adenocarcinoma-M/SMs & $5(55 \cdot 6)$ & $58(35.8)$ & \\
\hline Adenocarcinoma-SM/d & $1(11.1)$ & $15(9 \cdot 3)$ & \\
\hline \multicolumn{4}{|l|}{ Procedure-related factor } \\
\hline Procedural time, min & $90.7 \pm 58.6$ & $90.7 \pm 121.4$ & 0.30 \\
\hline Post-ESD ulcer appearance & & & 0.08 \\
\hline Clip closure & 0 & $52(32.1)$ & \\
\hline Coagulation & $9(100)$ & $110(67.9)$ & \\
\hline Visible vessel & $4(44 \cdot 4)$ & $64(39.5)$ & \\
\hline Non-visible vessel & $5(55 \cdot 5)$ & $46(28.4)$ & \\
\hline
\end{tabular}

Values are presented as mean \pm SD or number (\%).

LST-G, lateral spreading tumor-granular type; LST-NG, lateral spreading tumor-nongranular type; M/SMs, mucosa to superficial submucosa invasion (<1,000 $\mu \mathrm{m})$; SM/d, deep submucosa invasion ( $\geq 1,000 \mu \mathrm{m})$; ESD, endoscopic submucosal dissection.

in the SLE group. Regarding procedure-related factors, the clip closure rate was higher in the SLE group than in the no-SLE group (30.4\% vs. $20.6 \%, p=0.03$ ).

The total number of hemoclips used for clip closure in the SLE and no-SLE groups was $8.45 \pm 2.66$ and 8.24 \pm 3.84 , respectively (mean $\pm \mathrm{SD}, p=0.77$ ). In the coagulation group, ulcers with local hemostasis using endoclips were 42 cases in the SLE group and 28 cases in the noSLE group.

\section{SLE findings}

The incidence of high-risk ulcer stigma during SLE was $5.3 \%$ (9/171). Among patients with high-risk ulcer stigma, no Ia ulcers were observed during SLE, and $\mathrm{Ib}$ and IIa ulcers were observed in one and eight cases, respectively.

Patient-, lesion-, and procedure-related characteristics according to SLE findings are shown in Table 2. There was no significant association between patient-, lesion-, or procedure-related factors and the incidence of high-risk ulcer stigma during SLE. However, all of the post-ESD ulcers treated with clip closures remained 
Table 3. Second-look endoscopy findings according to the post-ESD ulcer appearance in coagulation group

\begin{tabular}{|c|c|c|}
\hline \multirow{2}{*}{ Forrest classification } & \multicolumn{2}{|c|}{ Post-ESD ulcer appearance in coagulation group, $\mathrm{n}(\%)$} \\
\hline & Visible vessel $^{\mathrm{a}}(\mathrm{n}=68)$ & Non-visible vessel ${ }^{\mathrm{b}}(\mathrm{n}=51)$ \\
\hline High-risk ulcer stigma & $4(5.9)$ & $5(9.8)$ \\
\hline Type Ib & $1(1.5)$ & 0 \\
\hline Type IIa & $3(4 \cdot 4)$ & $5(9.8)$ \\
\hline Low-risk ulcer stigma & $64(94.1)$ & $46(90.2)$ \\
\hline Type IIb & $9(13.2)$ & $9(17.6)$ \\
\hline Type IIc & $48(70.6)$ & $31(60.8)$ \\
\hline Type III & $7(10.3)$ & $6(11.8)$ \\
\hline
\end{tabular}

ESD, endoscopic submucosal dissection.

${ }^{\mathrm{a}}$ With remnant minor vessels.

${ }^{b}$ Without remnant minor vessels.

closed during SLE and were classified as having low-risk ulcer stigma. Thus, the proportion with coagulation tended to be higher in the high-risk ulcer stigma group than in the low-risk ulcer stigma group (100\% vs. 67.9\%, $p=0.08)$.

Table 3 shows the SLE findings according to the post-ESD ulcer appearance findings in the coagulation group. The incidence of high-risk ulcer stigma during SLE was not significantly different between the visible and non-visible vessels in the coagulation group (5.9\% vs. $9.8 \%, p=0.49$ ).

During SLE, endoscopic hemostasis was performed more frequently in the high-risk ulcer stigma group than in the low-risk ulcer stigma group (44.4\% [4/9] vs. $1.9 \%[3 / 162], p<0.001)$. In the high-risk ulcer stigma group, endoscopic hemostasis was performed with hemoclips in one patient with an oozing ulcer (type $1 b$ ). For type IIa ulcers $(\mathrm{n}=3)$, prophylactic hemostasis was performed with hemoclips $(n=1)$ and APC $(n=2)$.

\section{Incidence of delayed bleeding}

Post-ESD delayed bleeding occurred in three (o.8\%) patients, who belonged in the SLE group. In these three cases, all neoplasms were located on the rectum. Two patients with visible minor vessels on post-ESD ulcers developed delayed bleeding 2 days post-ESD. SLE findings were Forrest types IIa and IIb (Fig. 2), and prophylactic hemostasis was not performed during SLE in both patients. On the day of the delayed bleeding, they underwent endoscopic bleeding control with hemoclips and APC, and bleeding spots could not be retrospectively matched to dots on ulcers on SLE. Another patient underwent clip closure after completion of ESD and developed delayed bleeding 4 days post-ESD. During SLE, as the ulcer was closed and there was no evidence of bleeding, prophylactic hemostasis was not performed. However, when bleeding occurred, some clips were not present, and bleeding was observed in the partially opened ulcer. Bleeding control was successful via hemoclips. No death or admission to intensive care units occurred. Fig. 3 summarizes the post-ESD ulcer appearance, SLE findings using Forrest classification, and delayed bleeding occurrence.

\section{DISCUSSION}

In this study, the incidence of high-risk ulcer stigma during SLE was 5.3\% (9/171), and the tendency of coagulation to induce high-risk ulcer stigma was higher than that of clip closures in the post-ESD ulcer $(p=0.06)$. In the high-risk ulcer stigma group during SLE, only one patient underwent endoscopic hemostasis due to oozing ulcer (type $1 \mathrm{~b}$ ), while three patients underwent prophylactic hemostasis. Post-ESD delayed bleeding occurred in three (0.8\%) patients belonging to the SLE group, comprising one high-risk stigma patient and two low-risk stigma patients.

In our study, the rate of post-colorectal ESD bleeding was low at $0.8 \%$. In a systemic review of 22 studies that investigated the efficacy and safety of ESD for colorectal neoplasia [3], per-lesion bleeding rates were $2 \%$ (95\% CI, 

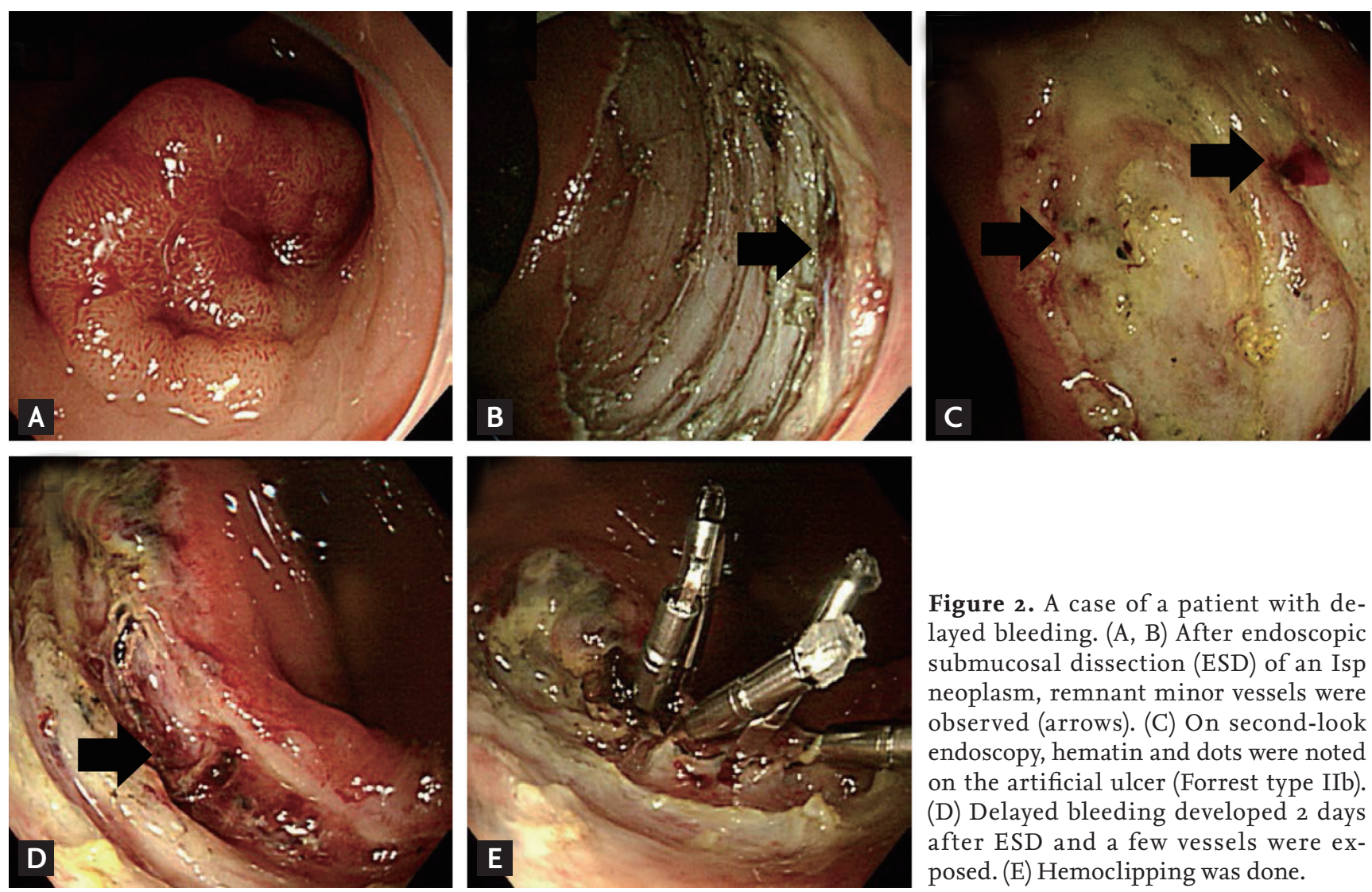

Figure 2. A case of a patient with delayed bleeding. (A, B) After endoscopic submucosal dissection (ESD) of an Isp neoplasm, remnant minor vessels were observed (arrows). (C) On second-look endoscopy, hematin and dots were noted on the artificial ulcer (Forrest type IIb). (D) Delayed bleeding developed 2 days after ESD and a few vessels were exposed. (E) Hemoclipping was done.

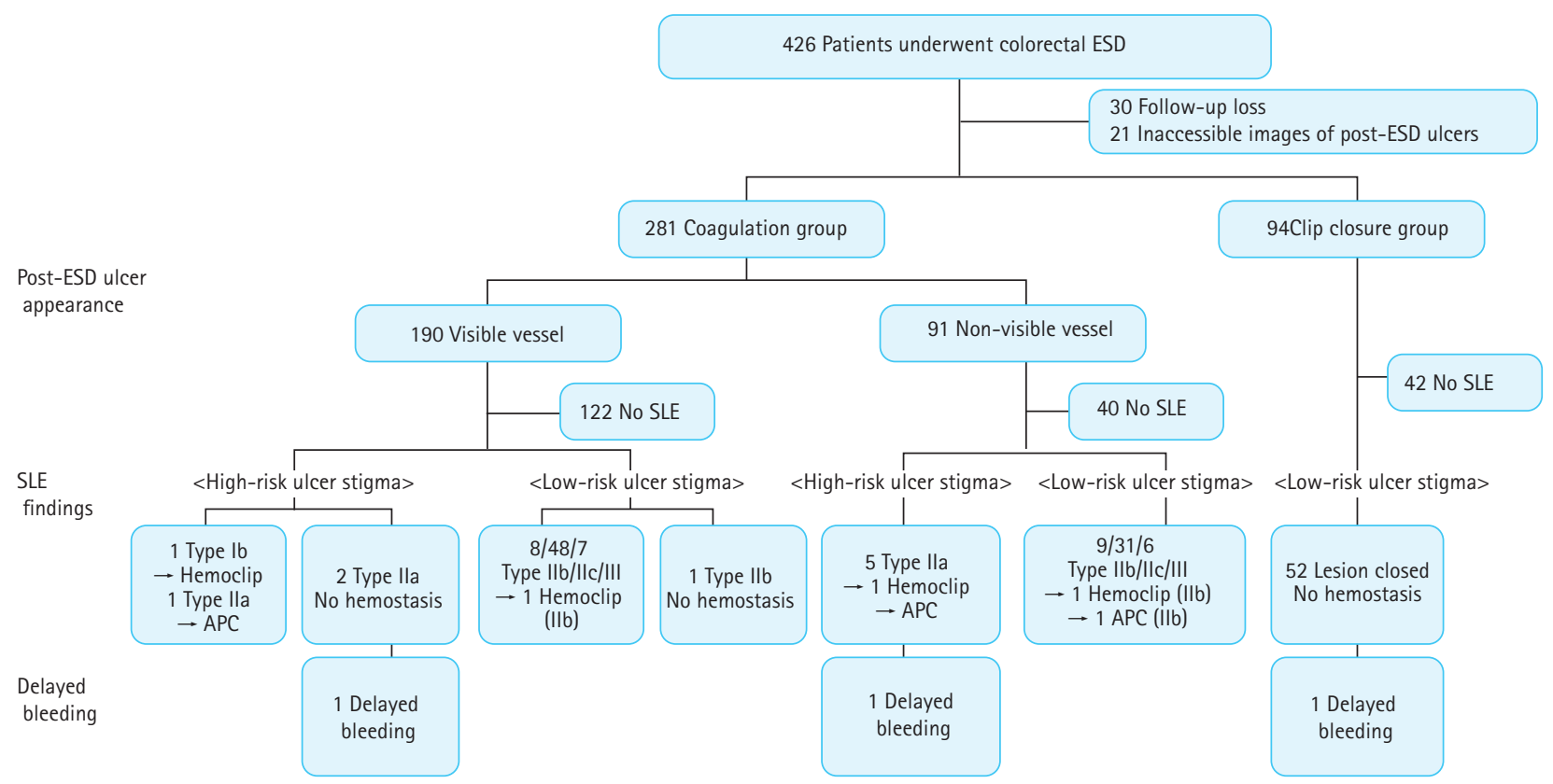

Figure 3. A summary of the post-endoscopic submucosal dissection (ESD) ulcer appearance, second-look endoscopy (SLE) findings using Forrest classification, and delayed bleeding occurrence. APC, argon plasma coagulation. 
$1 \%$ to $2 \%)$. However, they included one small study that showed exceptionally high bleeding rates (12\%) [20], and 17 studies reported post-ESD bleeding rates lower than $2 \%$, with nine of these studies reporting $0 \%$, which corresponded with our results. In this study, investigating the role of SLE in the prevention of delayed bleeding was limited, as the bleeding rate was low compared with the SLE and no-SLE groups, and baseline characteristics including lesion- and procedure-related factors were different between the SLE and no-SLE groups owing to our study's retrospective design.

However, SLEs performed after colorectal ESD may not be effective in preventing delayed bleeding due to multiple reasons. First, the incidence of high-risk ulcer stigma during SLE was as low as 5.3\%, of which only one underwent endoscopic hemostasis due to oozing-type bleeding, while the other three underwent prophylactic hemostasis. Although there was no bleeding in these three patients, it was uncertain whether SLE was helpful in preventing delayed bleeding in these patients. Previous gastric ESD studies reported that delayed post-ESD bleeding was more common in patients who underwent prophylactic hemostasis during SLE than in those who did not (odds ratio, $3.4 ; 95 \%$ confidence interval, 1.87 to 6.18) [16]. Second, all patients with post-ESD bleeding were in the SLE group. In the three cases with delayed bleeding, no bleeding was found during SLE and two cases showed low-risk stigma. None of them underwent hemostasis during SLE, and bleeding spots could not be retrospectively matched to dots on ulcers during SLE. In a study that investigated the efficacy of SLEs performed post-ESD for gastric epithelial neoplasm [21], bleeding occurred in 11 of 19 cases post-SLE, and bleeding spots could not be retrospectively matched to dots on ulcers during SLE in four cases.

In our results, among the coagulation group with post-ESD ulcer appearance, there were no differences between the visible vessel and non-visible vessel groups regarding the occurrence of high-risk ulcer stigma in SLE, but there were no delayed bleeding cases in the non-visible vessel group. Thus, prophylactic hemostasis of not only major vessels, but also minor vessels, on post-ESD ulcers after dissection might be helpful to prevent colorectal post-ESD bleeding $[7,22]$. However, as the muscularis propria of the colon is thinner and more easily perforated than the gastric mucosa, prophy- lactic hemostasis should be performed cautiously. In 94 (25.1\%) patients, prophylactic hemostasis was achieved by clip closure, and all remained closed during SLE. We classified the clip closure group as a low-risk ulcer stigma because we also considered closed artificial ulcers as important findings on SLE. This might have contributed to the low post-ESD bleeding rates. Prophylactic clipping of resection sites after endoscopic mucosal resections of large, flat, colorectal lesions is associated with a reduced incidence of delayed post-procedural bleeding [23]. In a small study that evaluated the efficacy and safety of prophylactic closures for large mucosal defects post-colorectal ESD, there were no bleeding in 18 cases of clip closures and nine cases of over-the-scope clip closures [24]. In our study, clip closures were maintained during SLE, but bleeding occurred in one case 4 days post-SLE. In this case, some clips were not present, and bleeding was observed in the partially opened ulcer. Thus, SLE might not be needed, particularly in cases of prophylactic clip closure.

This is the first study to investigate SLE findings after colorectal ESD. Although it is more complex than SLE performed after gastric ESD, large cases of post-colorectal ESD SLE were performed for both distal and proximal colon lesions. The incidence of high-risk ulcer stigma during SLE was low (5.3\%), and only one patient in the SLE group $(1 / 171,0.6 \%)$ underwent endoscopic hemostasis due to oozing bleeding to prevent delayed bleeding. Post-ESD delayed bleeding occurred in three (o.8\%) patients in the SLE group, belonging to the highrisk stigma and low-risk stigma groups.

This study has several limitations. First, due to its retrospective nature, the indications for performing SLE and prophylactic hemostasis during SLE were not consistent, which was dependent on the endoscopists' discretion. In addition, although the strategies of endoscopic hemostasis were based on the practical policy at our endoscopy center, we could not clearly control the hemostatic methods in all patients. Second, bleeding rates were relatively low, although this relatively low bleeding rate is probably a result of prophylactic coagulation and clip closure, and these hemostasis methods might be performed to prevent delayed bleeding. Owing to these two limitations, comparing delayed bleeding between the SLE and no-SLE groups was limited, and we focused on SLE findings, endoscopic hemostasis 
during SLE, and SLE findings of delayed bleeding cases. Further large prospective studies and more cases of bleeding are needed to evaluate the efficacy of SLE performed post-colorectal ESD. Third, we used the Forrest classification to evaluate SLE ulcer findings. Although the Forrest classification is a useful tool for planning endoscopic therapy for peptic ulcer bleeding and for evaluating gastric post-ESD ulcers, there are no specific classifications for colorectal ulcers due to the low incidence of colon ulcer and ulcer bleeding. In this study, the endoscopists tended to perform prophylactic hemostasis in high-risk ulcer stigma with Forrest classification during SLE. However, the incidence of delayed bleeding and prophylactic hemostasis in SLE was low and did not differ according to the risk of ulcer stigmata. Therefore, further studies investigating the classification of colorectal artificial ulcers are needed to evaluate the relationship between post-colorectal ESD ulcer appearance and various complications.

In conclusion, the incidence of high-risk ulcer stigma during SLE was low, and delayed bleeding occurred in the SLE cases of, both, high-risk and low-risk ulcer stigma groups. SLEs performed after colorectal ESD may not be effective in preventing delayed bleeding, and further prospective studies are needed to evaluate the efficacy of SLE in post-colorectal ESD.

\section{KEY MESSAGE}

1. The incidence of high-risk ulcer stigma during second-look endoscopy (SLE) was low in the patients who underwent colorectal endoscopic submucosal dissection (ESD).

2. SLE performed after colorectal ESD may not be effective in preventing delayed bleeding.

\section{Conflict of interest}

No potential conflict of interest relevant to this article was reported.

\section{Acknowledgments}

This study was supported by a National Research Foundation grant funded by the Korean government (2017R1A2B4006158) and the Soonchunhyang University Research Fund.

\section{REFERENCES}

1. Yamada M, Saito Y, Takamaru H, et al. Long-term clinical outcomes of endoscopic submucosal dissection for colorectal neoplasms in 423 cases: a retrospective study. Endoscopy 2017;49:233-242.

2. Shigita K, Oka S, Tanaka S, et al. Long-term outcomes after endoscopic submucosal dissection for superficial colorectal tumors. Gastrointest Endosc 2017;85:546-553.

3. Repici A, Hassan C, De Paula Pessoa D, et al. Efficacy and safety of endoscopic submucosal dissection for colorectal neoplasia: a systematic review. Endoscopy 2012;44:137-150.

4. Fuccio L, Hassan C, Ponchon T, et al. Clinical outcomes after endoscopic submucosal dissection for colorectal neoplasia: a systematic review and meta-analysis. Gastrointest Endosc 2017;86:74-86.

5. Jeong YH, Lee J, Kim SW, Seo GS, Kim HS, Joo YE. Clinicopathological feature and treatment outcome of patients with colorectal laterally spreading tumors treated by endoscopic submucosal dissection. Intest Res 2019;17:127134 .

6. Terasaki M, Tanaka S, Shigita K, et al. Risk factors for delayed bleeding after endoscopic submucosal dissection for colorectal neoplasms. Int J Colorectal Dis 2014;29:877882.

7. Suzuki S, Chino A, Kishihara T, et al. Risk factors for bleeding after endoscopic submucosal dissection of colorectal neoplasms. World J Gastroenterol 2014;20:18391845 .

8. Bahin FF, Naidoo M, Williams SJ, et al. Prophylactic endoscopic coagulation to prevent bleeding after wide-field endoscopic mucosal resection of large sessile colon polyps. Clin Gastroenterol Hepatol 2015;13:724-730.

9. Kim GU, Seo M, Song EM, et al. Association between the ulcer status and the risk of delayed bleeding after the endoscopic mucosal resection of colon. J Gastroenterol Hepatol 2017;32:1846-1851.

10. Elliott TR, Tsiamoulos ZP, Thomas-Gibson S, et al. Factors associated with delayed bleeding after resection of large nonpedunculated colorectal polyps. Endoscopy 2018;50:790-799.

11. Villanueva C, Balanzo J, Torras X, Soriano G, Sainz S, Vilardell F. Value of second-look endoscopy after injection therapy for bleeding peptic ulcer: a prospective and randomized trial. Gastrointest Endosc 1994;40:34-39.

12. Saeed ZA, Cole RA, Ramirez FC, Schneider FE, Hepps KS, 
Graham DY. Endoscopic retreatment after successful initial hemostasis prevents ulcer rebleeding: a prospective randomized trial. Endoscopy 1996;28:288-294.

13. Chiu PW, Lam CY, Lee SW, et al. Effect of scheduled second therapeutic endoscopy on peptic ulcer rebleeding: a prospective randomised trial. Gut 2003;52:1403-1407.

14. Ryu HY, Kim JW, Kim HS, et al. Second-look endoscopy is not associated with better clinical outcomes after gastric endoscopic submucosal dissection: a prospective, randomized, clinical trial analyzed on an as-treated basis. Gastrointest Endosc 2013;78:285-294.

15. Mochizuki S, Uedo N, Oda I, et al. Scheduled second-look endoscopy is not recommended after endoscopic submucosal dissection for gastric neoplasms (the SAFE trial): a multicentre prospective randomised controlled non-inferiority trial. Gut 2015;64:397-405.

16. Kim EH, Park SW, Nam E, Eun CS, Han DS, Park CH. Role of second-look endoscopy and prophylactic hemostasis after gastric endoscopic submucosal dissection: a systematic review and meta-analysis. J Gastroenterol Hepatol 2017;32:756-768.

17. Na S, Ahn JY, Choi KD, et al. Delayed bleeding rate according to the forrest classification in second-look endoscopy after endoscopic submucosal dissection. Dig Dis Sci 2015;60:3108-3117.

18. Tanaka S, Oka S, Chayama K. Colorectal endoscopic submucosal dissection: present status and future perspective, including its differentiation from endoscopic mucosal resection. J Gastroenterol 2008;43:641-651.

19. ASGE Standards of Practice Committee, Anderson MA, Ben-Menachem T, et al. Management of antithrombotic agents for endoscopic procedures. Gastrointest Endosc 2009;70:1060-1070.

20. Hurlstone DP, Atkinson R, Sanders DS, Thomson M, Cross SS, Brown S. Achieving Ro resection in the colorectum using endoscopic submucosal dissection. Br J Surg 2007;94:1536-1542.

21. Goto O, Fujishiro M, Kodashima S, et al. A second-look endoscopy after endoscopic submucosal dissection for gastric epithelial neoplasm may be unnecessary: a retrospective analysis of postendoscopic submucosal dissection bleeding. Gastrointest Endosc 2010;71:241-248.

22. Yoshida N, Yagi N, Naito Y, Yoshikawa T. Safe procedure in endoscopic submucosal dissection for colorectal tumors focused on preventing complications. World J Gastroenterol 2010;16:1688-1695.

23. Liaquat H, Rohn E, Rex DK. Prophylactic clip closure reduced the risk of delayed postpolypectomy hemorrhage: experience in 277 clipped large sessile or flat colorectal lesions and 247 control lesions. Gastrointest Endosc 2013;77:401-407.

24. Fujihara S, Mori H, Kobara H, et al. The efficacy and safety of prophylactic closure for a large mucosal defect after colorectal endoscopic submucosal dissection. Oncol Rep 2013;30:85-90. 

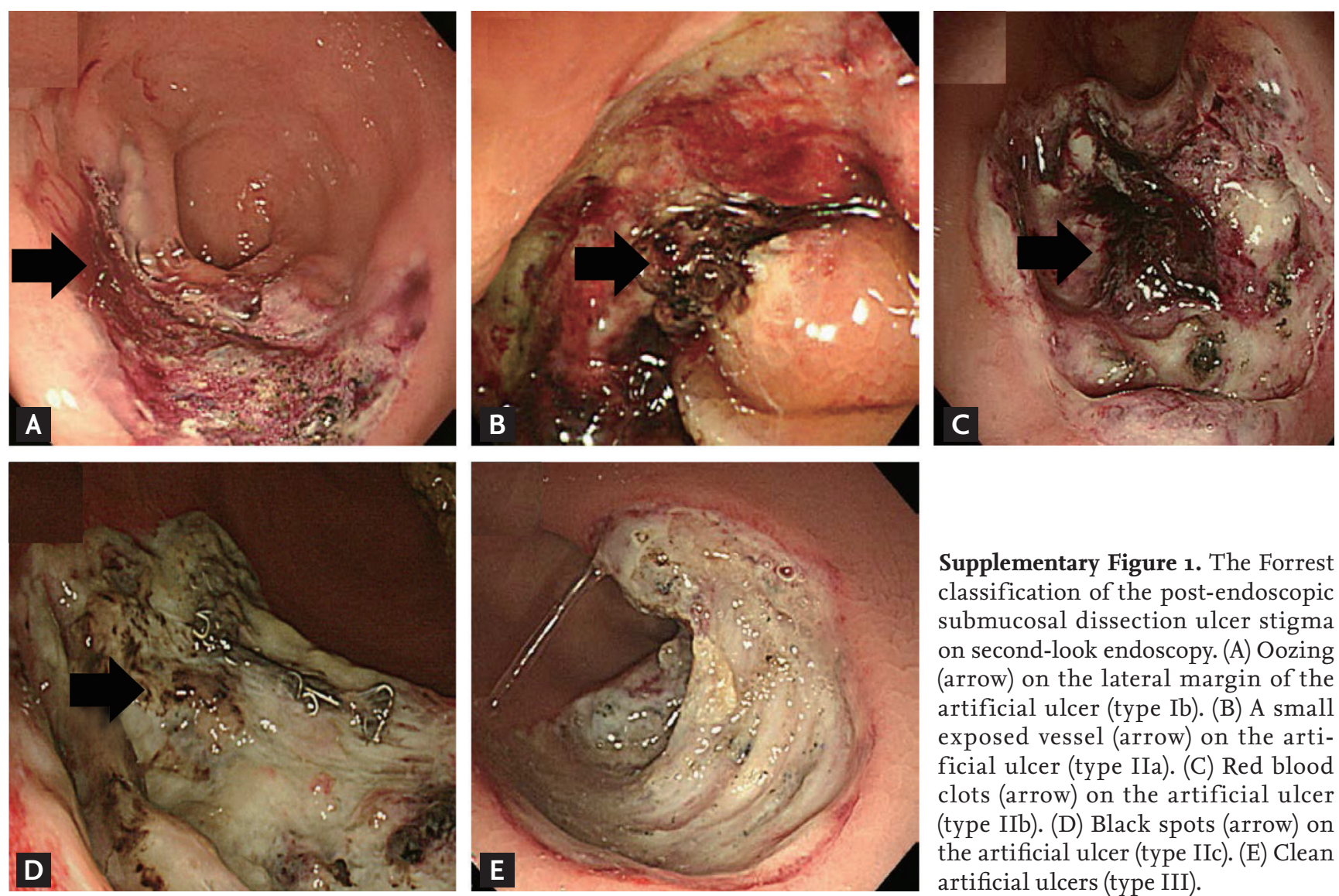

Supplementary Figure 1. The Forrest classification of the post-endoscopic submucosal dissection ulcer stigma on second-look endoscopy. (A) Oozing (arrow) on the lateral margin of the artificial ulcer (type Ib). (B) A small exposed vessel (arrow) on the artificial ulcer (type IIa). (C) Red blood clots (arrow) on the artificial ulcer (type IIb). (D) Black spots (arrow) on the artificial ulcer (type IIc). (E) Clean artificial ulcers (type III). 